\title{
ON SOME EXTENSIONS OF RELLICH'S INEQUALITY
}

\author{
B. G. PACHPATTE
}

\begin{abstract}
In this note we derive some new inequalities which in the special cases yields an extension of Rellich's inequality recently given by Bennett.
\end{abstract}

\section{Introduction}

In his fundamental work on perturbation theory of eigenvalue problems F. Rellich [9] established the following inequality.

Suppose that $u(x)$ is a function in $C_{0}^{\infty}\left(R^{n}-\{0\}\right)$ which is not identically zero, then

$$
\int_{R^{n}}|\Delta u|^{2} d x \geq \frac{n^{2}(n-4)^{2}}{16} \int_{R^{n}}|x|^{-4}|u|^{2} d x, \quad n \neq 2 .
$$

Rellich also proved this inequality for $n=2$ under some additional hypotheses (see, [3, p. 988]). Some important extensions of inequality (1) were established by Schmincke [10] in exploring selfadjointness criteria for a Schrödinger operator and Allegretto [2] in obtaining nonoscillation theorems for elliptic equations of order $2 n$. In a recent paper [3] Bennett established an interesting extension of Rellich's inequality by following Schmincke's method of proof. Despite the applications, we believe that the inequalities of the form (1) are of interest in their own right. The purpose of this note is to give further extensions of the inequality (1) which contains in the special cases an extension of (1) recently given by Bennett in [3]. The technique we will use to prove our results is based on the method employed by the authors in [10] and [3]. In fact, our search for further extensions of inequality (1) has led to some new inequalities which will allow for a broader range of applications.

\section{Statement of Results}

Throughout we assume that $H$ is an open, connected subset of $R^{n}$ that is not necessarily bounded, and that the boundary of $H, \partial H$, is sufficiently smooth in order that the Green formulas applies. A point in $R^{n}$ is denoted by $x=\left(x_{1}, \ldots, x_{n}\right)$ and its

Received September 17, 1990. 
norm is given by $|x|=\left(\sum_{i=1}^{n} x_{i}^{2}\right)^{\frac{1}{2}}$. We denote by $C^{m}(H)$ the vector space consisting of all functions $\phi$ which, together with all their partial derivatives $D^{\alpha} \phi$ of orders $|\alpha| \leq m$, are continuous on $H$ and denote by $C_{0}^{\infty}(H)$ the vector space of infinitely differentiable functions with compact support (see, $[1$, p. 9]).

We first establish the following Dubinskii type inequality [6, p. 168] which will be used in the proof of our main result.

Theorem 1. Let $p \geq 0, q \geq 1$ be constants; $g \in C^{2}(H), \triangle g \neq 0$ in $H$ and $u \in C_{0}^{\infty}(H)$ be real valued functions. Then

$$
\int_{H}|\Delta g||u|^{p+q} d x \leq(p+q)^{q} \int_{H}|\Delta g|^{-(q-1)}|\nabla g|^{q}|u|^{p}|\nabla u|^{q} d x,
$$

where $\nabla=\left(\frac{\partial}{\partial x_{1}}, \ldots, \frac{\partial}{\partial x_{n}}\right)$ and $\Delta=\frac{\partial^{2}}{\partial x_{1}^{2}}+\ldots+\frac{\partial^{2}}{\partial x_{n}^{2}}$.

The following version of the inequality (2) will be useful in our latter work.

Theorem 2. Let $p, q, g, u$ be as defined in Theorem. 1. Then

$$
\int_{H}|\Delta g||u|^{p+q} d x \leq(p+q)^{(p+q)} \int_{I}|\Delta g|^{-(p+q-1)}|\nabla g|^{p+q}|\nabla u|^{p+q} d x
$$

where $\nabla$ and $\Delta$ are as defined in Theorem 1.

Remark 1. If we take $g=|x|^{\alpha+2}, \alpha \geq 0$ is a real constant, and hence $|\nabla g|^{2}=$ $(\alpha+2)^{2}|x|^{2 \alpha+2}$ and $\Delta g=(\alpha+n)(\alpha+2)|x|^{\alpha}$ in inequality (3), then we get the following Hardy type inequality (see, [5, Lemma 12, p. 303])

$$
\int_{H}|x|^{\alpha}|u|^{p+q} d x \leq\left(\frac{p+q}{\alpha+n}\right)^{p+q} \int_{I}|x|^{p+q+\alpha}|\nabla u|^{p+q} d x .
$$

For different forms of the inequalities of the type (2)-(4), see $[7,8]$.

Our main result is given in the following theorem.

Theorem 3. If $p, q, g, u$ be as defined in. Theorem 1, then for any constants $\delta \geq 0, \epsilon>0$,

$$
\begin{aligned}
& \int_{H}|\Delta g|^{-(q-1)}|g|^{q}|u|^{p}|\Delta u|^{q} d x \\
\geq & -\epsilon^{q-1} q(p+q-1) \operatorname{sgn}(\Delta g) \int_{H} g|u|^{p+q-2}|\nabla u|^{2} d x \\
& -\epsilon^{q-1} \frac{q \delta}{p+q} \int_{H}|\Delta g|^{-(q-1)}|\nabla g|^{q}|u|^{p}|\nabla u|^{q} d x \\
& +\epsilon^{q-1}\left[\frac{q}{p+q}-(q-1) \epsilon+\frac{q \delta}{(p+q)^{q+1}}\right] \int_{H}|\Delta g||u|^{p+q} d x,
\end{aligned}
$$

where $\nabla$ and $\triangle$ are as defined in Theorem 1 . 
A slight variant of inequality (5) is embodied in the fcllowing theorem.

Theorem 4. If $p, q, g, u$ be as defined in Theorem 1 , then for any constants $\delta \geq 0, \epsilon>0$

$$
\begin{aligned}
& \int_{H}|\Delta g|^{-(p+q-1)}|g|^{p+q}|\Delta u|^{p+q} d x \\
\geq & -\epsilon^{(p+q-1)}(p+q)(p+q-1) \operatorname{sgn}(\Delta g) \int_{H} g|u|^{p+q-2}|\nabla u|^{2} d x \\
& -\epsilon^{(p+q-1)} \delta \int_{H}|\Delta g|^{-(p+q-1)}|\nabla g|^{p+q}|\nabla u|^{p+q} d x . \\
& +\epsilon^{(p+q-1)}\left[1-(p+q+-1) \epsilon+\frac{\delta}{(p+q)^{p+q}}\right] \int_{H}|\Delta g||u|^{p+q} d x,
\end{aligned}
$$

where $\nabla$ and $\triangle$ are as defined in Theorem 1 .

Remark 2. We note that in the special cases when $p=0, q=2$ and using the definition sgn $(\Delta g)=\frac{\Delta g}{|\Delta g|}$, inequalities (5) and (6) reduces to the following inequality

$$
\begin{aligned}
& \int_{H}|\Delta g|^{-1}|g|^{2}|\Delta u|^{2} d x \\
\geq & -\epsilon \int_{H}\left[2 g \Delta g+\delta|\nabla g|^{2}\right]|\Delta g|^{-1}|\nabla u|^{2} d x \\
& +\epsilon\left[1-\epsilon+\frac{\delta}{4}\right] \int_{H}|\Delta g||u|^{2} d x,
\end{aligned}
$$

which is recently established by Bennett [3, Theorem 5$]$. By taking $p=0$ and $q=4$ in (5) and $p=2, q=2$ in (6) we get an inequality which we believe is new to the literature. Furthermore, by specializing the conditions on $p, q$ and the function $g$ in (5) and (6) we get different inequalities of some interest in their own right and will have a broader range of applications.

\section{Proofs of Theorems 1 and 2}

By applying Green's first formula to $\int_{H} \Delta g|u|^{p+q} d x$, we have

$$
\int_{H} \Delta g|u|^{p+q} d x=-\int_{H} \nabla g \nabla\left(|u|^{p+q}\right) d x
$$

From (8) and using the definition $\operatorname{sgn}(\Delta g)=\frac{\Delta g}{|\Delta g|}$, the fact that $\nabla\left(|u|^{p+q}\right)=(p+$ $q)|u|^{p+q-1} \nabla u \operatorname{sgn} u$, and applying Hölder's inequality with indices $q, \frac{q}{q-1}$, we observe that 


$$
\begin{aligned}
\int_{H}|\Delta g||u|^{p+q} d x & =-\operatorname{sgn}(\Delta g) \int_{I} \nabla g \nabla\left(|u|^{p+q}\right) d x \\
& =-(p+q) \operatorname{sgn}(\Delta g) \int_{H} \nabla g|u|^{p+q-1} \nabla u \operatorname{sgn} u d x \\
& \leq(p+q) \int_{H}|\nabla g||u|^{p+q-1}|\nabla u| d x \\
& =(p+q) \int_{I I}\left[|\Delta g|^{-\left(\frac{q-1}{q}\right)}|\nabla g||u|^{\frac{p}{q}}|\nabla u|\right] \\
& \leq(p+q)\left\{\int_{H}|\Delta g|^{-(q-1)}|\nabla g|^{q}|u|^{p}|\nabla u|^{q} d x\right\}^{\frac{1}{q}} \\
& \cdot\left\{\int_{I I}|\Delta g||u|^{p+q} d x\right\}^{\frac{q-1}{q}} .
\end{aligned}
$$

If $\int_{I I}|\Delta g||u|^{p+q} d x=0$, then (2) is trivially true, otherwise we divide both sides of (9) by $\left\{\int_{H}|\Delta g||u|^{p+q} d x\right\}^{\frac{q-1}{q}}$ and then raise both sides of the resulting inequality to the power $q$ to get inequality (2). This complete the proof of Theorem 1 .

By following the above proof of Theorem 1, we have

$$
\begin{gathered}
\int_{I I}|\Delta g \| u|^{p+q} d x \leq(p+q) \int_{I I}|\nabla g||u|^{p+q-1}|\nabla u| d x \\
=(p+q) \int_{I I}\left[|\Delta g|^{-\left(\frac{p+q-1}{p+q}\right)} \mid \nabla g\|\nabla u\|\right] \\
\cdot\left[|\Delta g|^{\left(\frac{p+q-1}{p+q}\right)}|u|^{p+q-1}\right] d x .
\end{gathered}
$$

Now using the Hölder's inequality with indices $p+q, \frac{p+q}{p+q-1}$ on the right of (10) and following exactly the same arguments as in the last part of the proof of Theorem 1 given above with suitable changes we get the desired inequality in (3). The proof of Theorem 2 is complete.

Remark 3. If we take $g,|\nabla g|^{2}$ and $\Delta g$ as in Remark 1 in inequality (10) we get

$$
\begin{aligned}
& \int_{H}|x|^{\alpha}|u|^{p+q} d x \leq\left(\frac{p+q}{\alpha+n}\right) \int_{I I}|x|^{\alpha+1}|u|^{p+q-1}|\nabla u| d x \\
&=\left(\frac{p+q}{\alpha+n}\right) \int_{I}\left[|x|^{\frac{\alpha+1}{p+q}}|\nabla u|\right] \\
& \cdot\left[|x|^{-\left(\frac{\alpha+1}{p+q}\right)}|x|^{\alpha+1}|u|^{p+q-1}\right] d x .
\end{aligned}
$$

Using the IIölder's inequality with indices $p+q, \frac{p+q}{p+q-1}$ on the right of (11) we get the following Weyl type inequality (see, [5, Lemma 11, p. 303])

$$
\begin{array}{r}
\int_{H}|x|^{\alpha}|u|^{p+q} d x \leq\left(\frac{p+q}{\alpha+n}\right)\left\{\int_{H}|x|^{\alpha+1}|\nabla u|^{p+q} d x\right\}^{\frac{1}{p+q}} \\
\cdot\left\{\int_{H}|x|^{\alpha+1}|u|^{p+q} d x\right\}^{\frac{p+q-1}{p+q}} .
\end{array}
$$


For a version of Weyl's inequality in one independent variable, sce [4].

\section{Proofs of Theorems 3 and 4}

Let $A, B, C, D$ denote the integrals (without the exterior constants) in (5) successively. Applying Green's second formula to $\int_{I I} \Delta g|u|^{p+q} d x$ we have

$$
\int_{H} \Delta g|u|^{p+q} d x=\int_{I I} g \Delta\left(|u|^{p+q}\right) d x .
$$

Using the definition $\operatorname{sgn}(\Delta g)=\frac{\Delta g}{|\Delta g|}$ in (13) we obscrve that

$$
D=\operatorname{sgn}(\Delta g) \int_{I I} g \Delta\left(|u|^{p+q}\right) d x
$$

Using the fact that

$$
\begin{aligned}
\Delta\left(|u|^{p+q}\right)= & (p+q)|u|^{p+q-1} \Delta u \operatorname{sgn} u \\
& +(p+q)(p+q-1)|u|^{p+q-2}|\nabla u|^{2}
\end{aligned}
$$

in (14) we have

$$
\begin{aligned}
D= & \operatorname{sgn}(\Delta g)(p+q) \int_{I I} g|u|^{p+q-1} \Delta u \operatorname{sgn} u d x \\
& +\operatorname{sgn}(\Delta g)(p+q)(p+q-1) \int_{H} g|u|^{p+q-2}|\nabla u|^{2} d x \\
\leq & (p+q) \int_{I I}|g||u|^{p+q-1}|\Delta u| d x \\
& +(p+q)(p+q-1) \operatorname{sgn}(\Delta g) B \\
= & (p+q) \int_{I I}\left[|\Delta g|^{-\left(\frac{q-1}{q}\right)}|g||u|^{\frac{p}{q}}|\Delta u|\right] \\
& \cdot\left[|\Delta g|^{\left(\frac{q-1}{q}\right)}|u|^{p+q-1-\frac{p}{q}}\right] d x \\
& +(p+q)(p+q-1) \operatorname{sgn}(\Delta g) B .
\end{aligned}
$$

Now first applying the IÖlder's inequality with indices $q, \frac{q}{q-1}$ on the right side of (16) and then using the Young's inequality with indices $q, \frac{q}{q-1}$, we see that 


$$
\begin{aligned}
D \leq & (p+q)\left\{\int_{H}|\Delta g|^{-(q-1)}|g|^{q}|u|^{p}|\Delta u|^{q} d x\right\}^{\frac{1}{q}} \\
& \cdot\left\{\int_{H}|\Delta g||u|^{p+q} d z\right\}^{\frac{q-1}{q}} \\
& +(p+q)(p+q-1) \operatorname{sgn}(\Delta g) B \\
= & (p+q) A^{\frac{1}{q}} D^{\left(\frac{q-1}{q}\right)}+(p+q)(p+q-1) \operatorname{sgn}(\Delta g) B \\
= & (p+q)\left(\epsilon^{-\left(\frac{q-1}{q}\right)} A^{\frac{1}{q}}\right)\left(\epsilon^{\left(\frac{q-1}{q}\right)} D^{\left(\frac{q-1}{q}\right)}\right) \\
& +(p+q)(p+q-1) \operatorname{sgn}(\Delta g) B \\
\leq & \left(\frac{p+q}{q}\right) \epsilon^{-(q-1)} A+\frac{(p+q)(q-1)}{q} \epsilon D \\
& +(p+q)(p+q-1) \operatorname{sgn}(\Delta g) B
\end{aligned}
$$

for $\epsilon>0$. Now for any $\delta \geq 0$, from (2) we observe that

$$
\delta C-\frac{\delta}{(p+q)^{q}} D \geq 0 .
$$

Combining this fact with (17) we have

$$
\begin{aligned}
D \leq & \left(\frac{p+q}{q}\right) \epsilon^{-(q-1)} A+\frac{(p+q)(q-1)}{q} \epsilon D \\
& +(p+q)(p+q-1) \operatorname{sgn}(\Delta g) B+\delta C-\frac{\delta}{(p+q)^{q}} D
\end{aligned}
$$

for all $\epsilon>0$ and $\delta \geq 0$. Rewriting (18) we get the desired inequality in (5). This completes the proof of Theorem 3 .

In order to prove Theorem 4 , let $A, B, C, D$ denote the integrals (without the exterior constants) in (6) successively. By following the arguments in the first part of the proof of Theorem 3 we have

$$
\begin{aligned}
D \leq & (p+q) \int_{H}|g||u|^{p+q-1}|\Delta u| d x \\
& +(p+q)(p+q-1) \operatorname{sgn}(\Delta g) B \\
= & (p+q) \int_{H}\left[|\Delta g|^{-\left(\frac{p+q-1}{p+q}\right)}|g| \Delta u \mid\right] \\
& \cdot\left[|\Delta g|^{\left(\frac{p+q-1}{p+q}\right)}|u|^{p+q-1}\right] d x+(p+q)(p+q-1) \operatorname{sgn}(\Delta g) B .
\end{aligned}
$$

Now first using the Hölder's inequality with indices $p+q, \frac{p+q}{p+q-1}$, then Young's inequality with indices $p+q, \frac{p+q}{p+q-1}$ on the right in (19), the inequality (3) and following closely the arguments in the proof of Theorem 3 with suitable modifications we get the required
inequality in (6). The proof of Theorem 4 is complete. 
Remark 4. If we specialize the inequalities (5) and (6) by putting $g=|x|^{\alpha+2}, \alpha \geq$ 0 real and hence $|\nabla g|^{2}=(\alpha+2)^{2}|x|^{2 \alpha+2}, \Delta g=(\alpha+n)(\alpha+2)|x|^{\alpha}$, we get some new inequalities similar to that of inequality given by Bennett in [3, Corollary 6, p. 992]. For an interesting comparison of inequality in [3, Corollary 6] with the earlier extensions of Rellich's inequality (1) given by Allegretto [2] and Schmincke [10], see [3].

\section{References}

[1] R. A. Adams, "Sobolev spaces", Academic Press, New York, 1975.

[2] W. Allegretto, "Nonoscillation theory of elliptic equations of order $2 n$ ", Pacific J. Math. 64, 1-16, 1976.

[3] D. M. Bennett, “An extension of Rellich's inequality", Proc. Amer. Math. Soc. 106, 987-993, 1989.

[4] D. C. Benson, "Inequalities involving integrals of functions and their derivatives", J. Math. Anal. Appl. 17, 292-308, 1967.

[5] F. Bernis, "Compactness of the support for some nonlinear elliptic problems of arbitrary order in dimension N", Comm. Partial Diff. Eqs. 9, 271-312, 1984.

[6] J. A. Dubinskii, "Some integral inequalities and the solvaljility of degenerate quasilinear elliptic systems of diferential equations", Math. Sl. 64, 458-480, 1964.

[7] B. G. Pachpatte, "On Sobolev type integral inequalities", Proc. Royal. Soc. Edinburgh 103A, 1-14, 1986.

[8] B. G. Pachpatte, "A note on Dubinskii t.ype inequality", Libertas Mathematica 9, 49-57, 1989.

[9] F. Rellich, "Pcrturbation theory of eigenvalue problems", Gordon and Breach, New York, 1969.

[10] U. W. Schmincke, "Essential sclf-arljointness of a Schrödinger operator with strongly singular potential", Math. Z. 124, 47-50, 1972.

Department of Mathemalics and Statistics, Marallwadla University, A urangabad 431004 , (Maharashtra), India. 